\title{
Comparison of 3DCRT,VMAT and IMRT techniques in metastatic vertebra radiotherapy: A phantom Study
}

\author{
Sonay GEDIK, Sema TUNC, Arda KAHRAMAN, Sibel KAHRAMAN CETINTAS and Meral KURT
}

Uludag University, Medicine Faculty, Radiation Oncology Department, Bursa, Turkey

\begin{abstract}
Vertebra metastases can be seen during the prognosis of cancer patients. Treatment ways of the metastasis are radiotherapy, chemotherapy and surgery. Three-dimensional conformal therapy (3D-CRT) is widely used in the treatment of vertebra metastases. Also, Intensity Modulated Radiotherapy (IMRT) and Volumetric Arc Therapy (VMAT) are used too. The aim of this study is to examine the advantages and disadvantages of the different radiotherapy techniques. In the aspect of this goal, it is studied with a randophantom in Uludag University Medicine Faculty, Radiation Oncology Department. By using a computerized tomography image of the phantom, one 3DCRT plan, two VMAT and three IMRT plans for servical vertebra and three different 3DCRT plans, two VMAT and two IMRT plans for lomber vertebra are calculated. To calculate 3DCRT plans, CMS XiO Treatment System is used and to calculate VMAT and IMRT plans Monaco Treatment Planning System is used in the department. The study concludes with the dosimetric comparison of the treatment plans in the spect of critical organ doses, homogeneity and conformity index. As a result of this study, all critical organ doses are suitable for QUANTEC Dose Limit Report and critical organ doses depend on the techniques which used in radiotherapy. According to homogeneity and conformity indices, VMAT and IMRT plans are better than one in 3DCRT plans in servical and lomber vertebra radiotherapy plans.
\end{abstract}

\section{Introduction}

Treatment rate is increasing for elderly patients at 65 and more ages with the increasing population age today. It is expected that 70 percent of all cancer patients loses their lives in this age range [1]. It is estimated that this death rate will be doubled by 2030 [2,3]. Until 2050, 65 years of age and over are expected to receive four times more new cancer diagnoses [2,3]. Vertebral metastasis is an important morbidity source [4]. Vertebra is the most frequent site of body after lung and liver [5]. Approximately one-third of cancer patients develop bone metastasis, and 10 percent of these metastases are vertebra metastases [6]. For example, 10 percent of colorectal cancers have bone metastasis [7]. According to a study by Walther of 1948 on the autopsy of 3500 patients who lost their lives due to cancer, sixty six percent of all prostate cancers, fourty percent of breast cancer patients and thirty eight percent of thyroid cancer patients bone metastasis developed [8]. Patients with vertebral metastasis are palliative and treatment purpose is to control pain, to preserve or treat neurological functions, to prevent bladder involvement and to keep the patient standing [4]. The treatment of patients with vertebral metastases is decided by considering several factors.
These factors are; clinical data, duration of present symptoms, tumour type, radiosensitivity, tumour location, non-vertebral extension, vertebra length and medical status of the patient [4]. Techniques such as Intensity Modulated Radiotherapy (IMRT) and Volumetric Arc Therapy (VMAT) are also used in the radiotherapy of vertebral metastases, as well as conventional planning techniques. In 3-Dimensional Conformal Radiotherapy (3DCRT), the doses of healthy tissues and organs are high. The IMRT and VMAT methods allow the dose to accumulate in tumour volume, preserving normal tissues. This feature is very important for the patient in terms of the coming time. Nonetheless, it can lead to radiation-induced secondary cancers. The aim of this study is to answer the question of which treatment planning method is best suited to the application of radiotherapy and the most appropriate for critical organs in patients with vertebral metastases from 3DCRT, IMRT and VMAT treatment methods. 


\subsection{Treatment approaches to metastatic vertebras}

Bone metastases may be treated with external radiotherapy, radionuclides, surgery, chemotherapy and co-administration of one or more of the bisphosphonates [9].

Vertebra metastases are a common occurrence in the cancer prognosis. The methods of treatment are both radiotherapy or surgery or both of them. The use of 3DCRT in the radiotherapy of vertebra metastases is quite common. However, IMRT and VMAT methods can also be used.

3DCRT is a technique performed by matching radiation beams to tumour shape. Conventional external radiotherapy treatment is performed with the aid of a compensator or wedge to change the intensity or density of the radiation bundles at the single density throughout the treatment area. The treatment modality by changing the bundle density and obtaining a composite plan is called IMRT. VMAT is the application of IMRT (step and shoot and dynamic methods) where the treatment gantry unit is rotating with arcs. Treatment can be done with more than one arc or partial arc. In the literature, it is applied as $30 \mathrm{~Gy}$ with 10 fractions, 20 Gy with 5 fractions and 8 Gy with 1 fractions in world-wide metastatic vertebra radiotherapy.

\subsection{Materials and Reference Values}

\subsubsection{Randophantom}

The randophantom produced from tissue equivalent material consists of sections parallel to each other with a thickness of $2.5 \mathrm{~cm}$. These sections are connected to each other by removable inner support rods. Its section has pits closed to the bone equivalent to soft tissues or lung tissue. These holes are the designed so that the pits are removed and replaced with a dosimeter. Rando phantom represents a woman weighing $55 \mathrm{~kg}, 155 \mathrm{~cm}$ in length.

\subsubsection{Conformity and Homogeneity Indexes}

In the quality assessment of treatment plans, the homogeneity and conformity index formulas specified in the 83rd Report of ICRU (International Commission on Radiation Units and Measurements) were used [10].

$$
H I=\frac{D 2 \%-D 98 \%}{D 50 \%}
$$

D2\% : Doses of $2 \%$ of PTV volume

D98\%: Doses of $98 \%$ of PTV volume

D50\%: Doses of $50 \%$ of PTV volume

$$
C I=\frac{T V R I}{T V} x \frac{T V R I}{V R I}
$$

$\boldsymbol{T} \boldsymbol{V}_{\boldsymbol{R} \boldsymbol{I}}$ : Target volume receiving reference isodose $\boldsymbol{T V}$ : Target volume

$\boldsymbol{V}_{\boldsymbol{R}}$ : Total volume receiving reference isodose of the target volume and non-target volume
According to ICRU Report No. 83, if the homogeneity index value is close to 0 and the conformity index value is close to 1 , it is desirable to calculate the optimum treatment plan.

\subsubsection{Dose Limitations}

The dose-volume limits of QUANTEC (Quantitative Analysis of Normal Tissue Effects in the Clinic) were taken into account when determining critical organ doses.

\section{Techniques}

This study was carried out using randophantom belonging to Uludag University Medical Faculty, Radiation Oncology Department. Rando phantom was computerized tomography and computed with cervical and lumbar vertebral 3D-CRT, VMAT and IMRT treatment planning systems on this image. The total number of plans for both regions is 13 . The results obtained with these treatment plans are shown on the charts with the dose of the risky organs and the dose given to the target volunteer with the help of DVH.

The randophanton $\mathrm{CT}$ image was taken at a $3 \mathrm{~mm}$ slice thickness on the supine position in the head first position.

PTVs are created by $0.3 \mathrm{~cm}$ margins for CTV Cervical and CTV Lumber volumes. All VMAT plans were made with $6 \mathrm{MV}$ photons. In 3DCRT plans, both 6 $\mathrm{MV}$ and $15 \mathrm{MV}$ photons were used. It is applied as 30 Gy with 10 fractions for both servical and lumber vertebra in this study.

These treatment plans were calculated and abbreviations used to classify the data obtained by the calculated treatment plans are as follows:

S-3DCRT: Conformal radiotherapy treatment plan for cervical vertebra, calculated by using two treatment fields at right and left laterals

S-VMAT1: Radiotherapy treatment plan calculated by using two halves and one full arc beams for the cervical vertebra

S-VMAT2: Radiotherapy treatment plan for a cervical vertebra, calculated by using a full arc beam

S-IMRT1: IMRT treatment plan with seven treatment beams at $0,52,104,156,208,260,302$ degrees for cervical vertebra

S-IMRT2: IMRT treatment plan with seven treatment beams at 110, 130, 150, 180, 210, 230 and 250 degrees for the cervical vertebra

S-IMRT3: IMRT treatment plan with seven treatment beams at 110,130, 150,180, 210, 230 and 250 degrees for cervical vertebrae, 15 degree angle of collimator angle in 110 degree and 250 degree treatment areas.

L-3DCRT1: Conformal radiotherapy treatment plan calculated for the Lomber vertebra using AP and PA fields

L-3DCRT2: Conformal radiotherapy treatment plan for the Lomber vertebra, calculated by intervening AP and PA fields 
L-3DCRT3: Conformal radiotherapy treatment plan for Lomber vertebra, calculated by placing one AP and two posterior oblique treatment areas

L-VMAT1: Radiotherapy treatment plan calculated by using two half and one full arc beams for the lomber vertebra

L-VMAT2: Radiotherapy treatment plan for the lomber vertebra, calculated by using a full arc beam

L-IMRT1: IMRT treatment plan with seven treatment beams at $0,52,104,156,208,260,302$ degrees for the lomber vertebra

L-IMRT2: IMRT treatment plan with seven treatment beams at 110, 130, 150, 180, 210, 230 and 250 degrees for the lomber vertebra

\section{Results}

OAR doses calculated in treatment plans are shown in Table1 and Table 2.

Table 1. Risky organ doses in 3D-CRT and VMAT treatment plans for servical vertebra.

\begin{tabular}{|c|c|c|c|c|}
\hline \multicolumn{2}{|l|}{} & $\begin{array}{c}\text { S- } \\
\text { 3DCRT }\end{array}$ & $\begin{array}{c}\text { S- } \\
\text { VMAT1 }\end{array}$ & $\begin{array}{c}\text { S- } \\
\text { VMAT2 }\end{array}$ \\
\hline Thyroid & $\mathrm{V}_{26}$ & 13.9 & 22.79 & 25.83 \\
\hline Larynx & $\mathrm{D}_{\operatorname{mean}}$ (cGy) & 2743 & 2791 & 2811 \\
\hline OralCavity & $\mathrm{D}_{\operatorname{mean}}$ (cGy) & 112 & 1244 & 1350 \\
\hline Mandible & $\mathrm{D}_{\max }(\mathrm{cGy})$ & 3157 & 2899 & 2835 \\
\hline SpinalCord & $\mathrm{D}_{\max }$ (cGy) & 3092 & 3186 & 3206 \\
\hline
\end{tabular}

Table 2. Risky organ doses in IMRT treatment plans for servical vertebra.

\begin{tabular}{|c|c|c|c|c|}
\hline \multicolumn{2}{|c|}{} & $\begin{array}{c}\text { S- } \\
\text { IMRT1 }\end{array}$ & $\begin{array}{c}\text { S- } \\
\text { IMRT2 }\end{array}$ & $\begin{array}{c}\text { S- } \\
\text { IMRT3 }\end{array}$ \\
\hline Thyroid & $\mathrm{V}_{26}$ & 23.67 & 11.68 & 11.21 \\
\hline Larynx & $\mathrm{D}_{\text {mean }}(\mathrm{cGy})$ & 2753 & 2819 & 2820 \\
\hline OralCavity & $\mathrm{D}_{\operatorname{mean}}(\mathrm{cGy})$ & 1138 & 1477 & 1465 \\
\hline Mandible & $\mathrm{D}_{\max }(\mathrm{cGy})$ & 2939 & 2505 & 2575 \\
\hline SpinalCord & $\mathrm{D}_{\max }(\mathrm{cGy})$ & 3229 & 3225 & 3210 \\
\hline
\end{tabular}

Table 3. Risky organ doses in 3D-CRT treatment plans for lumber vertebra.

\begin{tabular}{|c|c|c|c|c|}
\hline & & $\begin{array}{c}\text { L- } \\
\text { 3DCRT1 }\end{array}$ & $\begin{array}{c}\text { L- } \\
\text { 3DCRT2 }\end{array}$ & $\begin{array}{c}\text { L- } \\
\text { 3DCRT3 }\end{array}$ \\
\hline \multirow{3}{*}{ Kidney(L) } & $\mathrm{V}_{12}$ & 22.18 & 19.69 & 28.5 \\
\cline { 2 - 5 } & $\mathrm{V}_{18}$ & 15.21 & 12.03 & 17.08 \\
\cline { 2 - 5 } & $\mathrm{V}_{20}$ & 13 & 9.61 & 14.14 \\
\hline \multirow{3}{*}{ Kidney(R) } & $\mathrm{V}_{12}$ & 24.61 & 19.47 & 29.67 \\
\cline { 2 - 5 } & $\mathrm{V}_{18}$ & 16.92 & 11.74 & 12.38 \\
\cline { 2 - 5 } & $\mathrm{V}_{20}$ & 14.5 & 9.38 & 8.51 \\
\hline Liver & $\mathrm{V}_{20}$ & 13.14 & 9.61 & 9 \\
\hline SpinalCord & $\mathrm{D}_{\max }(\mathrm{cGy})$ & 3212 & 3199 & 3339 \\
\hline
\end{tabular}

Table 4. Risky organ doses in VMAT and IMRT treatment plans for lumber vertebra.

\begin{tabular}{|c|c|c|c|c|c|}
\hline \multicolumn{2}{|c|}{} & $\begin{array}{c}\text { L- } \\
\text { VMAT1 }\end{array}$ & $\begin{array}{c}\text { L- } \\
\text { VMAT2 }\end{array}$ & $\begin{array}{c}\text { L- } \\
\text { IMRT1 }\end{array}$ & $\begin{array}{c}\text { L- } \\
\text { IMRT2 }\end{array}$ \\
\hline \multirow{3}{*}{$\begin{array}{c}\text { Kidney } \\
(\mathrm{L})\end{array}$} & $\mathrm{V}_{12}$ & 45.2 & 37.61 & 44.37 & 45.87 \\
\cline { 2 - 6 } & $\mathrm{V}_{18}$ & 17.9 & 15.99 & 19.92 & 22.53 \\
\cline { 2 - 6 } & $\mathrm{V}_{20}$ & 13.35 & 12.60 & 14.64 & 17.43 \\
\hline \multirow{2}{*}{$\begin{array}{c}\text { Kidney } \\
(\mathrm{R})\end{array}$} & $\mathrm{V}_{12}$ & 33.97 & 36.68 & 23.46 & 27.65 \\
\cline { 2 - 6 } & $\mathrm{V}_{18}$ & 16.15 & 16.53 & 10.24 & 13.4 \\
\cline { 2 - 6 } & $\mathrm{V}_{20}$ & 12.07 & 12.44 & 7.27 & 9.94 \\
\hline Liver & $\mathrm{V}_{20}$ & 0.7 & 0.57 & 0.66 & 0.33 \\
\hline \multirow{2}{*}{$\begin{array}{c}\text { Spinal } \\
\text { Cord }\end{array}$} & $\mathrm{D}_{\max }(\mathrm{cGy})$ & 3220 & 3243 & 3285 & 3282 \\
\hline \multirow{2}{*}{} & & & & & \\
\hline
\end{tabular}

\section{Conclusions}

Generally, the homogeneity index value is found to be the best in treatment planning calculated by VMAT and IMRT techniques.

The optimal conformity index value in cervical vertebra treatment planning was obtained by treatment plans calculated by VMAT and IMRT techniques. All plans are consistent with this limitation given the dose limits for the thyroid described in QUANTEC. But the most appropriate treatment plan is the S-IMRT3 treatment plan, according to the V26 dose. The S3DCRT and S-IMRT2 treatment plans have a close thyroid dose with the S-IMRT3 treatment plan. It is thought that the best result of thyroid V26 in IMRT treatment plans is caused by gantry and collimator angles where the treatment fields used in S-IMRT2 and S-IMRT3 treatment plans are formed. In the S-3DCRT treatment plan, the treatment areas were created from the right and left laterals, allowing for thyroid protection.

In cervical vertebra radiotherapy, spinal cord dose is very important. According to the dose limits stated in QUANTEC, the maximum dose of spinal cord should be below 45 Gy. If the dose prescribed in all plans is below $45 \mathrm{~Gy}$, the most appropriate treatment plan for this dose limitation was obtained with the S-3DCRT treatment plan. While the S-3DCRT treatment plan followed the maximum dose S-VMAT1 treatment plan received by the spinal cord, there were no significant differences between the other VMAT and IMRT treatment plans.

Significant differences were observed between treatment plans in terms of MU per fraction. It has been observed that the MU value per fraction seen in treatment plans calculated with the 3DCRT technique is about half of the value seen in treatment plans calculated by VMAT and IMRT technique. It has been observed that VMAT treatment plans are higher in terms of MU value per fraction seen in treatment plans calculated with the IMRT technique.

The best homogeneity index value was obtained from the lumbar vertebra treatment plan calculated by VMAT technique. The treatment plans calculated by the VMAT technique are followed by the treatment plans calculated by the IMRT technique. The highest 
homogeneity index value was calculated in the L3DCRT3 treatment plan.

The homogeneity index value in treatment planning, which is calculated for the lumbar vertebra, is ideal for treatment planning computed by the VMAT and IMRT technique.

Conformity index value is above 1 value for all treatment plans calculated for the lumber vertebra. Conformity index values are obtained from treatment plans obtained with the best IMRT technique in order to be close to 1 value.

\section{References}

1. R.Yancik, I. Ries, Hematological Oncology Clinic North America, 14:17-23 (2000).

2. J.A. Hayman, K.M. Langa, M.U. Kabeto, J ClinicOncol 19:3219;-3225 (2001)

3. A. Jatoi, S. Hillman, P. Stella, J Clin Oncol 23:9113-9119 (2005)

4. D.R. Fourney, Z.L. Gökaslan, Neurosurgery Clinic doi:10.1012/j.nec.2004.04.008 (2004)

5. D. Lubgan, A. Ziegaus, DOI:10.1007/s00066-014-0780-4

6. D.A. Wong, V.L. Fornasier, I. MacNab 15:1-4 (1990)

7. D. Santini, Ann Oncol ;23:2072-7 (2012)

8. H.E. Walther, Bens Schwabe Verlag (1948)

9. Kemik Metastazları,Temel ve Klinik Radyoterapi Kitabı,Türk Radyasyon Onkolojisi Derneği,s.387.

10. ICRU Report 83: Prescribing ,Recording,adn Recording Photon-Beam Intensity Modulated Arc Therapy,Vol.10,No:1 (2010) 